\title{
Comprehensive analysis of competing endogenous RNA networks associated with cholangiocarcinoma
}

\author{
FANGTING XU ${ }^{1 *}$, YUCHONG ZHAO $^{2 *}$, GANG QIN $^{1}$, YE HUAN $^{1}$, LONGYAN LI $^{1}$ and WEI GAO ${ }^{1}$ \\ ${ }^{1}$ Department of Anesthesia, Xiangya Hospital, Central South University, Changsha, Hunan 410008; \\ ${ }^{2}$ Department of Gastroenterology and Hepatology, Tongji Hospital, Tongji Medical College, \\ Huazhong University of Science and Technology, Wuhan, Hubei 430030, P.R. China
}

Received January 14, 2019; Accepted August 12, 2019

DOI: $10.3892 /$ etm.2019.8052

\begin{abstract}
Cholangiocarcinoma (CCA) is the second most common type of primary malignancy of the liver. Certain long non-coding RNAs (lncRNAs) have been demonstrated to have key roles in tumor pathogenesis by binding to microRNAs (miRNAs). However, the competing endogenous RNA (ceRNA) network of CCA remains to be fully determined. In the present study, the RNA expression profiles for CCA were downloaded from The Cancer Genome Atlas and further analyzed. A total of 318 differentially expressed (DE) lncRNAs, 87 DE miRNAs and 3,851 DE mRNAs were identified from 36 CCA samples and 9 adjacent non-tumor samples (for lncRNAs and miRNAs, fold change $\geq 2.5$ and $\mathrm{P}<0.01$; for $\mathrm{mRNAs}$, fold change $\geq 2$ and $\mathrm{P}<0.01$ ). Further bioinformatics analyses were performed and the ceRNA network for CCA was constructed, which included 16 lncRNAs, 55 miRNAs and 373 mRNAs. Survival analysis of all genes in the network revealed that high expression of the $\mathrm{mRNAs}$ fucosyltransferase $4(\mathrm{P}<0.005)$ and huntingtin-interacting protein 1 related $(\mathrm{P}<0.001)$ has a positive impact on the overall survival of patients with CAA. Furthermore, the lncRNAs H19 and PVT1, and the miRNAs Homo sapiens (hsa)-miR-16-5p and hsa-miR-424-5p, together with peroxisome proliferator-activated receptors, may also have important roles in the pathogenesis of CCA. The present study provided data to further the understanding of and research into the molecular mechanisms implicated in CCA.
\end{abstract}

\section{Introduction}

Cholangiocarcinoma (CCA) is a deadly malignancy of the biliary tree. Due to the high frequency of diagnosis at a late stage, metastasis and recurrence, surgical treatment for CCA

Correspondence to: Dr Wei Gao, Department of Anesthesia, Xiangya Hospital, Central South University, 87 Xiangya Road, Changsha, Hunan 410008, P.R. China

E-mail: 342152143@qq.com

*Contributed equally

Key words: competing endogenous RNAs, long-noncoding RNA, H19, PVT1, cholangiocarcinoma, peroxisome proliferator-activated receptor is associated with poor outcomes. The 5-year survival rate is $5-10 \%$ in patients with CCA (1). Therefore, understanding the molecular mechanisms involved in the tumorigenesis of CCA is a critical step for improving early diagnosis, reducing mortality, and developing effective targeted therapies. Long non-coding RNAs (lncRNAs) are autonomously transcribed non-coding RNAs of $>200 \mathrm{nt}$ in length, which have an important role in the regulation of gene transcription through the recruitment of chromatin-modifying enzymes (2). Accumulating studies have indicated that IncRNAs may interact with microRNAs (miRNAs/miRs) as competing endogenous RNAs (ceRNAs), and regulate the expression of target genes, which may have a role in tumor occurrence and progression (3). Therefore, lncRNAs are considered to be potential diagnostic and prognostic biomarkers of malignancy $(4,5)$. Based on these facts, Salmena et al (3) provided the ceRNA hypothesis and constructed a large-scale ceRNA regulatory network, which may explain tumor processes and present opportunities for novel therapies.

In previous studies, regulatory ceRNA networks composed of lncRNAs, miRNAs and mRNAs have been used to study molecular mechanisms of tumor occurrence and progression. Numerous studies have indicated that ceRNA regulatory lncRNA-miRNA-mRNA networks are implicated in the occurrence and progression of gastric, breast, pancreatic and liver cancer (6-9). Recently, IncRNA actin filament associated protein 1 (AFAP1)-antisense (AS)1 was reported to promote the growth and metastasis of CAA (10). AFAP1-AS1 expression was upregulated in CCA tumor samples and its knockdown reduced cell stress filament integrity, suggesting that it may be a diagnostic and prognostic biomarker for CCA. Another study indicated that the expression of IncRNA colon cancer-associated transcript 1 (CCAT1) was significantly upregulated in CAA samples, and promoted cell migration and invasion by suppressing miR-152 (11). Based on the aforementioned studies, it may be hypothesized that dysregulation of certain IncRNAs may promote CAA by regulating key pathways. Therefore, identification of a CAA-associated ceRNA network may be useful for understanding the role of ceRNAs in the genesis of CAA and therapeutic outcomes (12). In the present study, a ceRNA network of CAA was constructed, including 16 lncRNAs, 55 miRNAs and 373 mRNAs. Based on the network, survival analysis of all genes suggested that 
fucosyltransferase 4 (FUT4) and huntingtin-interacting protein 1 related (HIP1R) were associated with overall survival. These results provided further insight into the mechanisms of the pathogenesis of CAA, and may provide potential novel therapeutic markers for CAA treatment.

\section{Materials and methods}

Patients and The Cancer Genome Atlas (TCGA) data retrieval. The microarray data for 45 CCA samples were obtained from TCGA data portal (https://portal.gdc.cancer.gov/), with search results up to October 14th, 2018 included. The RNA and miRNA sequencing (seq) data were obtained from the IlluminaHiseq RNASeq and the IlluminaHiSeq_miRNASeq sequencing platforms. All data are open access and free to download. The sequencing data included the corresponding RNA-seq and miRNA-seq data. The human samples were divided into 2 groups: The CCA samples $(n=36)$ and adjacent non-tumor samples $(n=9)$. All of the protocols were in accordance with the guidelines of TCGA and no further ethical approval was required, since all of the data were collected from TCGA (https://cancergenome.nih. gov/publications/publicationguidelines).

Identification of differentially expressed (DE) IncRNAs, miRNAs and miRNAs. The raw data of the microarray datasets were preprocessed via background correction and normalization. Prior to the analysis, all unexpressed RNAs were filtered out by using R language (version 3.2.5; https://cran.r-project. org/). According to the R language results, those genes with mean read count $\leq 1$ were deleted. The lncRNAs and mRNAs were identified using the Ensembl database (version 89; http://www.ensembl.org/index.html). Subsequently, the DE mRNAs, lncRNAs and miRNAs of the two groups were obtained using edgeR software (13). The false discovery rate (FDR) was used for multiple comparisons of statistically significant P-values. The fold change (FC) was used for measuring the differential expression levels of genes, where $\mid \log 2 \mathrm{FCl} \geq 2$ and $\mathrm{FDR}$ adjusted to $\mathrm{P}<0.01$ were considered to indicate a significant DE mRNA or IncRNA; the standard for miRNAs was a fold change $\geq 2.5$ and an FDR adjusted to $\mathrm{P}<0.01$. Finally, all of the DE RNAs were analyzed and a volcano map was generated using the $\mathrm{R}$ platform.

Generation of the ceRNA regulatory network of CCA. To investigate the association between ceRNAs in patients with CAA, the lncRNA-miRNA-mRNA regulatory network was constructed, which was established through the following procedures based on the results of the DE analysis. First, the regulatory interactions between IncRNAs and miRNAs were predicted using the miRcode database (14). Subsequently, miRNA-targeted mRNAs were extracted from the miRTarBase (15), miRDB (16) and TargetScan (17) databases. Finally, using Cytoscape 3.5.1 (http://www.cytoscape.org/), the ceRNA regulatory network was generated and visualized.

Functional enrichment analysis. In order to better understand the mechanisms of CCA tumorigenesis, Gene Ontology (GO) and Kyoto Encyclopedia of Genes and Genomes (KEGG) pathway analyses of DE mRNAs were performed using the Database for Annotation, Visualization and Integrated

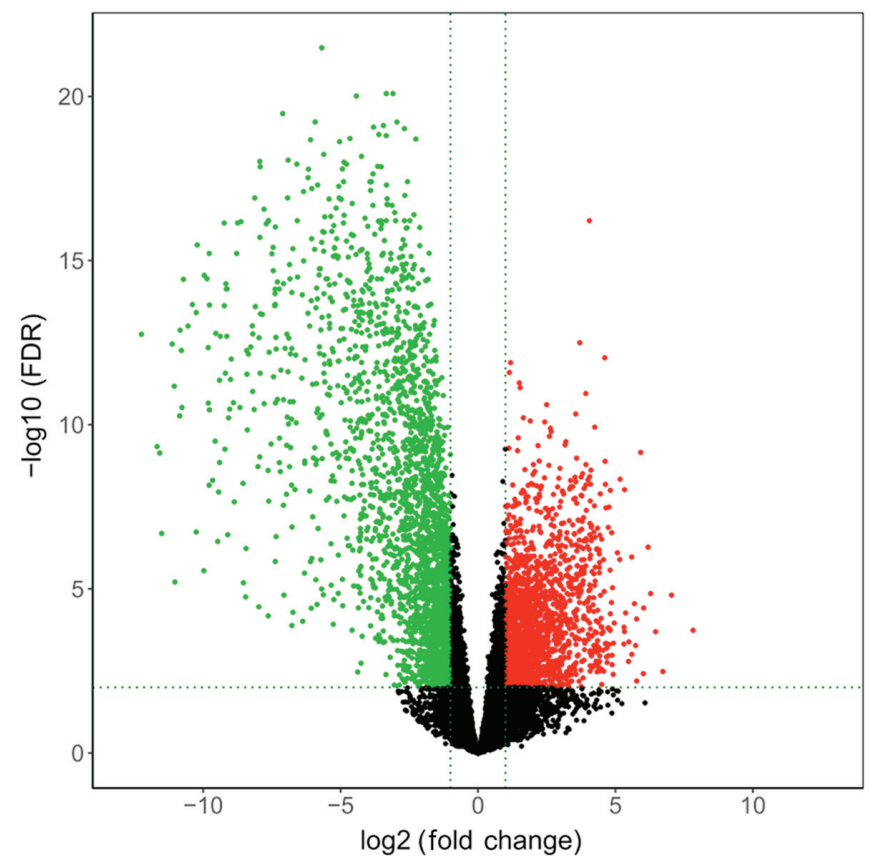

Figure 1. Volcano plot displaying the distribution of differentially expressed mRNAs. Green spots represent downregulated genes and red spots represent upregulated genes. FDR, false discovery rate.

Discovery (DAVID; http://www.david.abcc.ncifcrf.gov/). A GO term or KEGG pathway with FDR $<0.05$ was considered statistically significant. The enriched GO terms and pathways of the DE mRNAs with the most significant P-values were ranked by their enrichment score (-log P-value).

Survival analysis. The R survival package (version 2.41-3; https://CRAN.R-project.org/package=survival) was used for the survival analysis. The Kaplan-Meier method was used to estimate cumulative survival rates and the log-rank test was then used to compare the differences in overall survival between the different groups. $\mathrm{P}<0.05$ was considered to indicate statistical significance.

\section{Results}

DE lncRNAs, mRNAs and miRNAs in CCA. The present study investigated the differential RNA expression in 36 CCA tissues and 9 adjacent non-tumor tissues. The integrated analysis identified 318 DE lncRNAs, 3,851 DE mRNAs and 87 DE miRNAs using the edgeR package. Of the $318 \mathrm{DE}$ IncRNAs, 205 were upregulated (64.5\%) and 113 (35.5\%) were downregulated. A total of 1,549 (40.2\%) mRNAs were identified to be upregulated and 2,302 (59.8\%) were downregulated out of the 3,851 DE mRNAs. For the 87 DE miRNAs, 41 (47.1\%) were upregulated and the remaining $46(52.9 \%)$ were downregulated. The top 20 upregulated and downregulated lncRNAs, mRNAs and miRNAs are listed in Tables I-III, respectively. In addition, the distributions of all DE mRNAs are presented in a volcano plot in Fig. 1.

Construction of a ceRNA regulatory network in CCA. To better understand the characteristics of the lncRNAs and to further clarify the interactions between the IncRNAs and 
Table I. The top 20 up- and downregulated lncRNAs (ranked by P-values).

A, Top 20 upregulated lncRNAs

\begin{tabular}{|c|c|c|c|}
\hline Ensembl ID & $\log \mathrm{FC}$ & P-value & FDR \\
\hline ENSG00000261659 & 3.198316385 & $1.55 \times 10^{-11}$ & $3.30 \times 10^{-10}$ \\
\hline ENSG00000253210 & 2.985738665 & $1.21 \times 10^{-10}$ & $2.13 \times 10^{-09}$ \\
\hline ENSG00000261183 & 4.001322806 & $2.92 \times 10^{-10}$ & $4.81 \times 10^{-09}$ \\
\hline ENSG00000228109 & 3.369141828 & $3.07 \times 10^{-10}$ & $5.02 \times 10^{-09}$ \\
\hline ENSG00000265688 & 3.446807939 & $4.91 \times 10^{-10}$ & $7.64 \times 10^{-09}$ \\
\hline ENSG00000261068 & 4.766573772 & $2.45 \times 10^{-09}$ & $3.24 \times 10^{-08}$ \\
\hline ENSG00000172965 & 2.575378258 & $3.51 \times 10^{-09}$ & $4.50 \times 10^{-08}$ \\
\hline ENSG00000273230 & 2.560329476 & $4.77 \times 10^{-09}$ & $5.97 \times 10^{-08}$ \\
\hline ENSG00000234741 & 2.018672373 & $1.11 \times 10^{-08}$ & $1.30 \times 10^{-07}$ \\
\hline ENSG00000261801 & 3.691796251 & $1.53 \times 10^{-08}$ & $1.74 \times 10^{-07}$ \\
\hline ENSG00000285255 & 4.217290775 & $1.65 \times 10^{-08}$ & $1.86 \times 10^{-07}$ \\
\hline ENSG00000226711 & 2.650291782 & $3.45 \times 10^{-08}$ & $3.56 \times 10^{-07}$ \\
\hline ENSG00000261437 & 4.32349391 & $6.22 \times 10^{-08}$ & $6.02 \times 10^{-07}$ \\
\hline ENSG00000277283 & 1.914981951 & $1.10 \times 10^{-07}$ & $1.01 \times 10^{-06}$ \\
\hline ENSG00000243479 & 5.595465468 & $1.18 \times 10^{-07}$ & $1.08 \times 10^{-06}$ \\
\hline ENSG00000244041 & 1.90448619 & $1.32 \times 10^{-07}$ & $1.20 \times 10^{-06}$ \\
\hline ENSG00000269680 & 3.185294307 & $1.55 \times 10^{-07}$ & $1.39 \times 10^{-06}$ \\
\hline ENSG00000273759 & 2.435273695 & $1.66 \times 10^{-07}$ & $1.47 \times 10^{-06}$ \\
\hline ENSG00000257556 & 2.979912742 & $1.96 \times 10^{-07}$ & $1.71 \times 10^{-06}$ \\
\hline ENSG00000255381 & 1.597506825 & $2.13 \times 10^{-07}$ & $1.85 \times 10^{-06}$ \\
\hline
\end{tabular}

B, Top 20 downregulated lncRNAs

\begin{tabular}{|c|c|c|c|}
\hline Ensembl ID & $\log \mathrm{FC}$ & P-value & FDR \\
\hline ENSG00000225756 & -4.439300193 & $5.12 \times 10^{-18}$ & $6.55 \times 10^{-16}$ \\
\hline ENSG00000251165 & -5.214981183 & $6.12 \times 10^{-17}$ & $5.09 \times 10^{-15}$ \\
\hline ENSG00000261572 & -3.875298917 & $1.23 \times 10^{-15}$ & $6.95 \times 10^{-14}$ \\
\hline ENSG00000263400 & -4.480906234 & $1.29 \times 10^{-15}$ & $7.23 \times 10^{-14}$ \\
\hline ENSG00000215386 & -3.687757377 & $1.76 \times 10^{-15}$ & $9.49 \times 10^{-14}$ \\
\hline ENSG00000264575 & -2.497423285 & $2.22 \times 10^{-15}$ & $1.17 \times 10^{-13}$ \\
\hline ENSG00000267390 & -3.142413979 & $2.31 \times 10^{-15}$ & $1.20 \times 10^{-13}$ \\
\hline ENSG00000234456 & -3.123344646 & $5.80 \times 10^{-15}$ & $2.68 \times 10^{-13}$ \\
\hline ENSG00000261012 & -7.173215127 & $6.10 \times 10^{-14}$ & $2.19 \times 10^{-12}$ \\
\hline ENSG00000235609 & -2.872714774 & $1.54 \times 10^{-13}$ & $4.97 \times 10^{-12}$ \\
\hline ENSG00000261578 & -4.233216461 & $1.78 \times 10^{-13}$ & $5.66 \times 10^{-12}$ \\
\hline ENSG00000228794 & -1.905394222 & $8.41 \times 10^{-13}$ & $2.31 \times 10^{-11}$ \\
\hline ENSG00000259370 & -4.369092089 & $3.65 \times 10^{-12}$ & $8.74 \times 10^{-11}$ \\
\hline ENSG00000215256 & -2.110042475 & $3.88 \times 10^{-12}$ & $9.20 \times 10^{-11}$ \\
\hline ENSG00000223797 & -2.072567868 & $5.02 \times 10^{-12}$ & $1.17 \times 10^{-10}$ \\
\hline ENSG00000275494 & -2.27756684 & $1.10 \times 10^{-11}$ & $2.41 \times 10^{-10}$ \\
\hline ENSG00000269386 & -2.361574508 & $2.36 \times 10^{-11}$ & $4.81 \times 10^{-10}$ \\
\hline ENSG00000273616 & -2.942297335 & $3.18 \times 10^{-11}$ & $6.25 \times 10^{-10}$ \\
\hline ENSG00000267675 & -5.990122102 & $5.41 \times 10^{-11}$ & $1.03 \times 10^{-09}$ \\
\hline ENSG00000260274 & -1.989229751 & $5.54 \times 10^{-11}$ & $1.05 \times 10^{-09}$ \\
\hline
\end{tabular}

lncRNA, long non-coding RNA; ID, identification; FC, fold change; FDR, false discovery rate.

miRNAs, an IncRNA-miRNA-mRNA-associated regulatory network was constructed. First, the 318 DE lncRNAs derived from the miRcode database were used; these were applied to the Perl program, which identified 56 pairs of interacting 
Table II. The top 20 up- and down-regulated mRNAs (ranked by P-values).

A, Top 20 upregulated mRNAs

\begin{tabular}{|c|c|c|c|}
\hline Ensembl ID & $\log \mathrm{FC}$ & P-value & FDR \\
\hline ENSG00000067225 & 4.055367983 & $2.56996 \times 10^{-19}$ & $6.10345 \times 10^{-17}$ \\
\hline ENSG00000167642 & 3.701088077 & $7.00796 \times 10^{-15}$ & $3.17737 \times 10^{-13}$ \\
\hline ENSG00000166145 & 4.613180178 & $2.32377 \times 10^{-14}$ & $9.14954 \times 10^{-13}$ \\
\hline ENSG00000080603 & 1.181826005 & $3.47222 \times 10^{-14}$ & $1.28677 \times 10^{-12}$ \\
\hline ENSG00000129351 & 1.139806521 & $7.36096 \times 10^{-14}$ & $2.57927 \times 10^{-12}$ \\
\hline ENSG00000127616 & 1.503804232 & $1.66381 \times 10^{-13}$ & $5.31921 \times 10^{-12}$ \\
\hline ENSG00000102317 & 1.536020573 & $2.42826 \times 10^{-13}$ & $7.47564 \times 10^{-12}$ \\
\hline ENSG00000186765 & 3.918721626 & $3.7811 \times 10^{-13}$ & $1.11584 \times 10^{-11}$ \\
\hline ENSG00000149639 & 2.497348198 & $8.96987 \times 10^{-13}$ & $2.46252 \times 10^{-11}$ \\
\hline ENSG00000272398 & 3.551356872 & $1.82259 \times 10^{-12}$ & $4.72611 \times 10^{-11}$ \\
\hline ENSG00000179051 & 1.650897428 & $2.44245 \times 10^{-12}$ & $6.15218 \times 10^{-11}$ \\
\hline ENSG00000112118 & 1.902906765 & $3.13164 \times 10^{-12}$ & $7.63689 \times 10^{-11}$ \\
\hline ENSG00000164221 & 2.428188979 & $3.41876 \times 10^{-12}$ & $8.25024 \times 10^{-11}$ \\
\hline ENSG00000139734 & 4.246825211 & $5.12237 \times 10^{-12}$ & $1.18639 \times 10^{-10}$ \\
\hline ENSG00000166557 & 2.635202192 & $5.60838 \times 10^{-12}$ & $1.28503 \times 10^{-10}$ \\
\hline ENSG00000106012 & 2.659102098 & $7.13445 \times 10^{-12}$ & $1.60899 \times 10^{-10}$ \\
\hline ENSG00000034510 & 2.598284344 & $1.03483 \times 10^{-11}$ & $2.27358 \times 10^{-10}$ \\
\hline ENSG00000115561 & 1.462267513 & $1.14603 \times 10^{-11}$ & $2.51053 \times 10^{-10}$ \\
\hline ENSG00000187642 & 3.168721529 & $1.99786 \times 10^{-11}$ & $4.13444 \times 10^{-10}$ \\
\hline ENSG00000130702 & 2.181520136 & $2.11404 \times 10^{-11}$ & $4.35678 \times 10^{-10}$ \\
\hline
\end{tabular}

B, Top 20 downregulated mRNAs

\begin{tabular}{|c|c|c|c|}
\hline Ensembl ID & $\log \mathrm{FC}$ & P-value & FDR \\
\hline ENSG00000080709 & -5.686469859 & $2.2178 \times 10^{-26}$ & $3.31827 \times 10^{-22}$ \\
\hline ENSG00000105607 & -3.33705124 & $1.59062 \times 10^{-24}$ & $8.19986 \times 10^{-21}$ \\
\hline ENSG00000059769 & -3.096941246 & $1.64414 \times 10^{-24}$ & $8.19986 \times 10^{-21}$ \\
\hline ENSG00000155666 & -4.429426553 & $2.5976 \times 10^{-24}$ & $9.71631 \times 10^{-21}$ \\
\hline ENSG00000143257 & -7.106918488 & $1.12249 \times 10^{-23}$ & $3.35895 \times 10^{-20}$ \\
\hline ENSG00000091831 & -5.926384811 & $2.4347 \times 10^{-23}$ & $6.04595 \times 10^{-20}$ \\
\hline ENSG00000115841 & -2.951203464 & $2.82861 \times 10^{-23}$ & $6.04595 \times 10^{-20}$ \\
\hline ENSG00000160323 & -3.440732637 & $4.13922 \times 10^{-23}$ & $7.74137 \times 10^{-20}$ \\
\hline ENSG00000120158 & -3.801441126 & $5.20254 \times 10^{-23}$ & $8.64894 \times 10^{-20}$ \\
\hline ENSG00000205707 & -2.678156335 & $6.41175 \times 10^{-23}$ & $9.59327 \times 10^{-20}$ \\
\hline ENSG00000133027 & -3.608581255 & $1.0559 \times 10^{-22}$ & $1.43621 \times 10^{-19}$ \\
\hline ENSG00000119673 & -3.336686032 & $1.26151 \times 10^{-22}$ & $1.57289 \times 10^{-19}$ \\
\hline ENSG00000118777 & -4.659839514 & $1.66311 \times 10^{-22}$ & $1.91412 \times 10^{-19}$ \\
\hline ENSG00000179152 & -2.262072095 & $1.86674 \times 10^{-22}$ & $1.99501 \times 10^{-19}$ \\
\hline ENSG00000117009 & -6.093754472 & $2.11114 \times 10^{-22}$ & $2.1058 \times 10^{-19}$ \\
\hline ENSG00000213398 & -5.036902246 & $2.55547 \times 10^{-22}$ & $2.38968 \times 10^{-19}$ \\
\hline ENSG00000116761 & -5.616242901 & $6.69097 \times 10^{-22}$ & $5.88884 \times 10^{-19}$ \\
\hline ENSG00000109929 & -4.242818901 & $8.11342 \times 10^{-22}$ & $6.74406 \times 10^{-19}$ \\
\hline ENSG00000168306 & -6.910609036 & $1.11334 \times 10^{-21}$ & $8.76728 \times 10^{-19}$ \\
\hline ENSG00000124713 & -7.940751186 & $1.28333 \times 10^{-21}$ & $9.6006 \times 10^{-19}$ \\
\hline
\end{tabular}

ID, identification; FC, fold change; FDR, false discovery rate.

lncRNAs and miRNAs. From the 87 DE miRNAs retrieved, it was predicted that 55 of them were able to interact with 16 DE lncRNAs. mRNAs targeted by these 55 DE miRNAs were selected in all three databases (miRTarBase, miRDB and TargetScan). The targeted mRNAs with DE miRNAs retrieved from the miRcode database were cross-checked. The $373 \mathrm{DE}$ 
Table III. The top 20 up- and downregulated miRNAs (ranked by P-values).

A, Top 20 upregulated miRNAs

\begin{tabular}{|c|c|c|c|}
\hline miRNA & $\log \mathrm{FC}$ & P-value & FDR \\
\hline hsa-miR-183-5p & 4.836903706 & $6.38 \times 10^{-17}$ & $1.40 \times 10^{-14}$ \\
\hline hsa-miR-182-5p & 4.483302702 & $6.43 \times 10^{-17}$ & $1.40 \times 10^{-14}$ \\
\hline hsa-miR-21-5p & 2.485307292 & $6.28 \times 10^{-14}$ & $6.52 \times 10^{-12}$ \\
\hline hsa-miR-96-5p & 5.172417117 & $1.81 \times 10^{-13}$ & $1.31 \times 10^{-11}$ \\
\hline hsa-miR-27a-3p & 1.975273987 & $3.22 \times 10^{-13}$ & $1.99 \times 10^{-11}$ \\
\hline hsa-miR-34c-3p & 3.911770715 & $2.54 \times 10^{-10}$ & $4.76 \times 10^{-09}$ \\
\hline hsa-miR-222-3p & 2.267762864 & $3.08 \times 10^{-10}$ & $5.34 \times 10^{-09}$ \\
\hline hsa-miR-23a-3p & 1.494080752 & $4.43 \times 10^{-10}$ & $7.12 \times 10^{-09}$ \\
\hline hsa-miR-34c-5p & 4.181782951 & $1.10 \times 10^{-08}$ & $1.45 \times 10^{-07}$ \\
\hline hsa-miR-92b-3p & 2.769232681 & $1.75 \times 10^{-08}$ & $2.23 \times 10^{-07}$ \\
\hline hsa-miR-330-5p & 1.866060112 & $2.30 \times 10^{-08}$ & $2.78 \times 10^{-07}$ \\
\hline hsa-miR-454-3p & 1.608154597 & $2.67 \times 10^{-08}$ & $3.13 \times 10^{-07}$ \\
\hline hsa-miR-181d-5p & 2.43995135 & $3.65 \times 10^{-08}$ & $4.17 \times 10^{-07}$ \\
\hline hsa-let-7e-5p & 1.391443094 & $4.99 \times 10^{-08}$ & $5.42 \times 10^{-07}$ \\
\hline hsa-miR-221-3p & 1.752600485 & $8.05 \times 10^{-08}$ & $8.27 \times 10^{-07}$ \\
\hline hsa-miR-181b-5p & 1.952571231 & $9.66 \times 10^{-08}$ & $9.26 \times 10^{-07}$ \\
\hline hsa-miR-200b-3p & 2.634730289 & $9.82 \times 10^{-08}$ & $9.26 \times 10^{-07}$ \\
\hline hsa-miR-24-2-5p & 1.597889448 & $1.14 \times 10^{-07}$ & $1.05 \times 10^{-06}$ \\
\hline hsa-miR-301a-3p & 1.537288068 & $2.14 \times 10^{-07}$ & $1.94 \times 10^{-06}$ \\
\hline hsa-miR-99b-3p & 1.648708541 & $2.45 \times 10^{-07}$ & $2.13 \times 10^{-06}$ \\
\hline
\end{tabular}

B, Top 20 downregulated miRNAs

\begin{tabular}{|c|c|c|c|}
\hline miRNA & $\log \mathrm{FC}$ & P-value & FDR \\
\hline hsa-miR-148a-3p & -2.8329 & $5.07 \times 10^{-15}$ & $7.33 \times 10^{-13}$ \\
\hline hsa-miR-4662a-5p & -3.27785 & $7.51 \times 10^{-14}$ & $6.52 \times 10^{-12}$ \\
\hline hsa-miR-101-3p & -2.08208 & $4.85 \times 10^{-13}$ & $2.63 \times 10^{-11}$ \\
\hline hsa-miR-505-3p & -2.01987 & $2.01 \times 10^{-12}$ & $9.68 \times 10^{-11}$ \\
\hline hsa-miR-378a-3p & -3.24209 & $3.83 \times 10^{-12}$ & $1.66 \times 10^{-10}$ \\
\hline hsa-miR-148a-5p & -2.53305 & $7.56 \times 10^{-12}$ & $2.98 \times 10^{-10}$ \\
\hline hsa-miR-378a-5p & -2.94829 & $1.74 \times 10^{-11}$ & $6.30 \times 10^{-10}$ \\
\hline hsa-miR-125b-2-3p & -2.4123 & $2.24 \times 10^{-11}$ & $7.48 \times 10^{-10}$ \\
\hline hsa-miR-483-3p & -4.78428 & $2.72 \times 10^{-11}$ & $8.43 \times 10^{-10}$ \\
\hline hsa-miR-122-3p & -7.01304 & $3.26 \times 10^{-11}$ & $9.43 \times 10^{-10}$ \\
\hline hsa-miR-194-3p & -3.2353 & $3.93 \times 10^{-11}$ & $1.07 \times 10^{-09}$ \\
\hline hsa-miR-139-3p & -2.76339 & $5.01 \times 10^{-11}$ & $1.28 \times 10^{-09}$ \\
\hline hsa-let-7c-5p & -2.24221 & $6.46 \times 10^{-11}$ & $1.56 \times 10^{-09}$ \\
\hline hsa-miR-99a-3p & -2.34474 & $8.98 \times 10^{-11}$ & $1.97 \times 10^{-09}$ \\
\hline hsa-miR-675-3p & -5.17096 & $9.09 \times 10^{-11}$ & $1.97 \times 10^{-09}$ \\
\hline hsa-miR-139-5p & -3.13233 & $1.08 \times 10^{-10}$ & $2.24 \times 10^{-09}$ \\
\hline hsa-miR-378c & -2.82711 & $2.22 \times 10^{-10}$ & $4.38 \times 10^{-09}$ \\
\hline hsa-miR-885-5p & -6.05348 & $2.63 \times 10^{-10}$ & $4.76 \times 10^{-09}$ \\
\hline hsa-miR-99a-5p & -2.41861 & $3.84 \times 10^{-10}$ & $6.42 \times 10^{-09}$ \\
\hline hsa-miR-483-5p & -4.28222 & $4.93 \times 10^{-10}$ & $7.65 \times 10^{-09}$ \\
\hline
\end{tabular}

miR/miRNA, microRNA; hsa, Homo sapiens; FC, fold change; FDR, false discovery rate. 


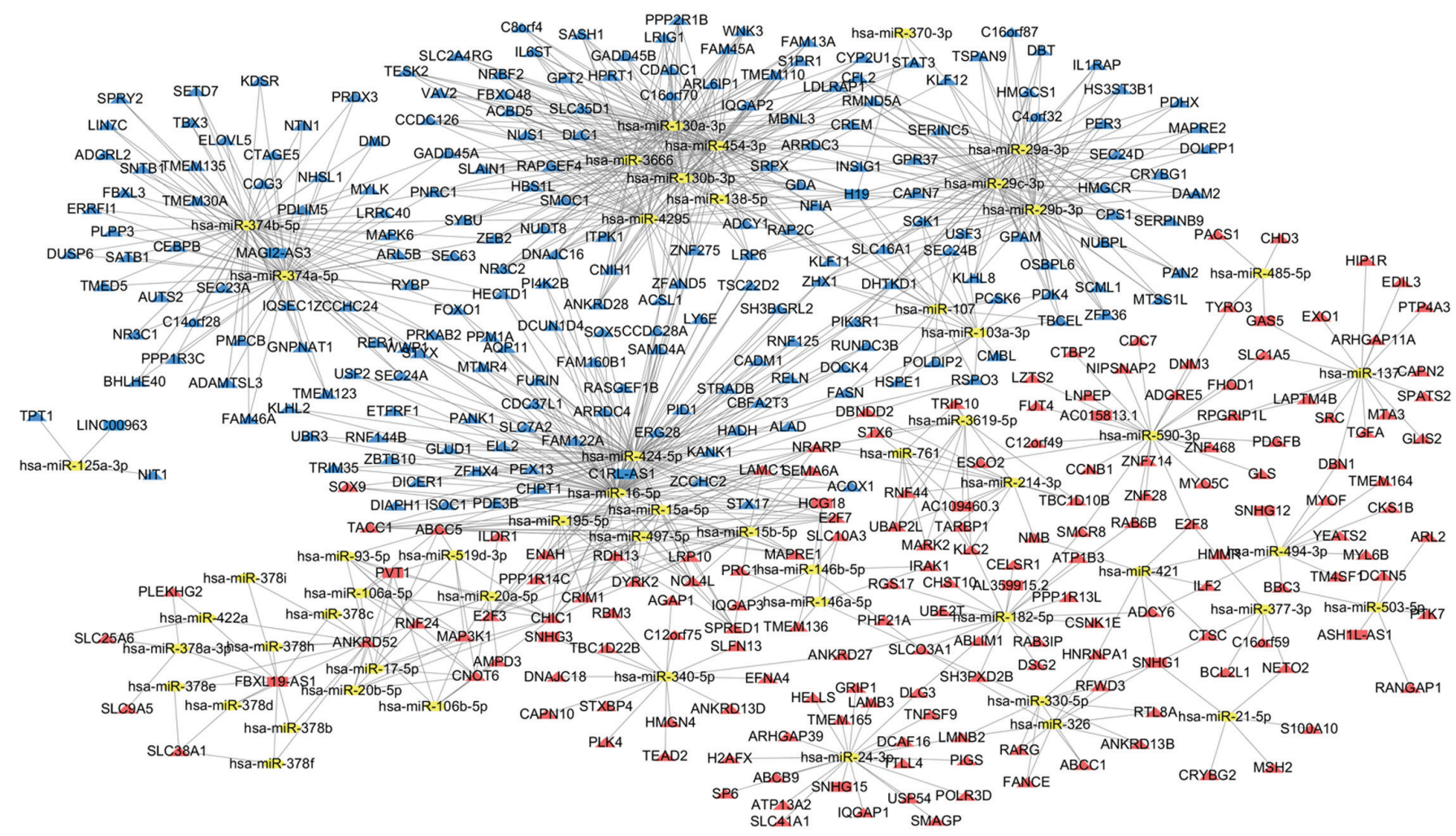

Figure 2. Competing endogenous RNA regulatory network in cholangiocarcinoma. The squares represent long non-coding RNAs, triangles indicate mRNAs and yellow circles represent miRs. Blue indicates downregulated RNAs and red represents upregulated RNAs. miR, microRNA; Hsa, Homo sapiens.

targeted mRNAs were then selected. Finally, the ceRNA regulatory network for CCA was created by incorporating $16 \mathrm{DE}$ lncRNAs, 55 DE miRNAs and 373 DE mRNAs (Fig. 2).

Functional enrichment analysis. GO analysis revealed that there were 427 enriched GO terms with statistical significance in the category biological process (BP), 44 in the category cellular component (CC) and 105 in the category molecular function (MF). The top enriched terms for BP, CC and MF are small molecule catabolic process, mitochondrial matrix and coenzyme binding, respectively (Fig. 3). KEGG analysis revealed 49 pathways associated with mRNAs, including 'cell metabolism', 'proliferation' and 'sustained angiogenesis'. The top 15 pathways are provided in Fig. 4 and most of them are associated with metabolism.

Survival analysis and the ceRNA network. To identify the prognostic characteristics of the ceRNA network, each gene of the 16 lncRNAs, 55 miRNAs and 373 mRNAs were analyzed using Kaplan-Meier survival analysis separately. The results revealed that only two DE mRNAs in the network (FUT4 and HIP1R) were associated with overall survival. The Kaplan-Meier survival analysis indicated that high levels of FUT4 $(\mathrm{P}<0.005)$ and HIP1R $(\mathrm{P}<0.001)$ were positively associated with overall survival (Fig. 5).

\section{Discussion}

CCA is the second most common type of primary liver malignancy. Although surgical resection remains the only potential treatment, it is frequently unfeasible due to the advanced tumor stage at the initial diagnosis, with a 5-year survival rate of $<5-10 \%$ (18). Diagnostic and therapeutic biomarkers are urgently required for patients with CCA. In recent years, accumulating evidence has indicated that IncRNAs have a key role in cancer. It has been suggested that lncRNAs may serve as sponges for miRNA, reducing their regulatory effect on mRNAs (19). This function introduces an extra layer of complexity in the miRNA-target interaction network, the dysregulation of which may contribute to the development and progression of multiple diseases (19). Although recent studies have reported that certain lncRNAs, including AFAP1-AS1, CCAT1, nuclear paraspeckle assembly transcript 1 and metastasis associated lung adenocarcinoma transcript 1 , may be associated with CCA, it remains necessary to perform a more comprehensive analysis of the ceRNA regulatory networks in CCA (20).

The present study systematically analyzed and constructed a ceRNA regulatory network containing 16 DE lncRNAs, 55 DE miRNAs and 373 DE mRNAs, in an attempt to better understand the pathogenesis of CCA. In this network, the lncRNAs primarily bound to Homo sapiens (hsa)-miR-16-5p, hsa-miR-424-5p, hsa-miR-130a-3p, hsa-miR-130b-3p and hsa-miR-454-3p. In addition, hsa-miR-16-5p and hsa-miR-424-5p were the largest nodes that interacted with 82 non-coding and coding RNAs in the current network, suggesting that these two miRNAs may be critical to the pathogenesis of CCA. The enriched GO terms of the DE mRNAs were strongly linked to metabolism and included 'small molecule catabolic process', 'organic acid catabolic process' and 'carboxylic acid catabolic process'. Pathway analysis revealed that 'carbon metabolism', 'peroxisome proliferator-activated receptor (PPAR) signaling pathway', 'bile secretion' and 'fat 


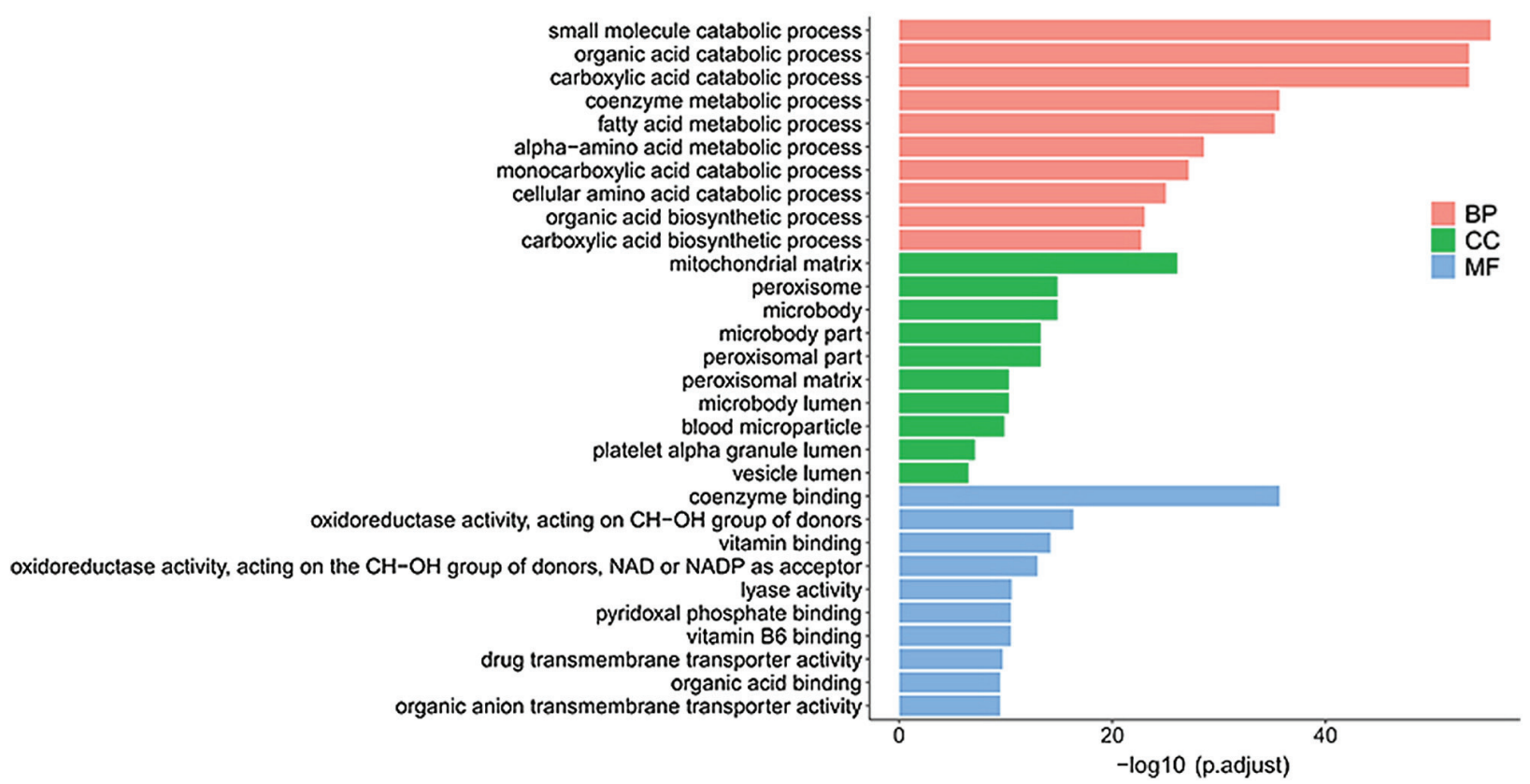

Figure 3. Functional enrichment analysis of differentially expressed mRNAs. The top 10 significantly enriched Gene Ontology terms in the categories BP, CC and $\mathrm{MF}$ are provided. $\mathrm{BP}$, biological process; $\mathrm{CC}$, cellular component; MF, molecular function.

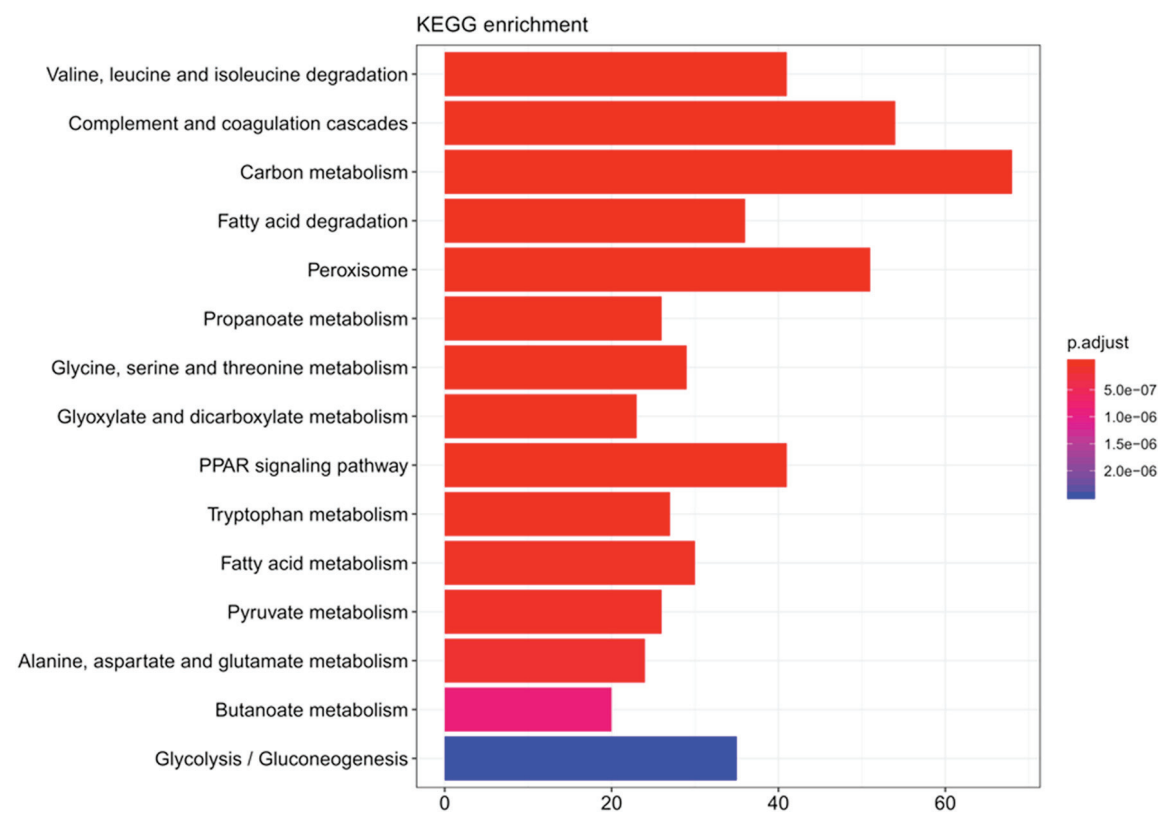

Figure 4. The top 15 KEGG pathways with the most significant P-values. The x-axis represents the number of differentially expressed mRNAs involved in the respective pathway. KEGG, Kyoto Encyclopedia of Genes and Genomes; PPAR, peroxisome proliferator-activated receptor.

digestion' were significantly enriched, and these have been reported to exert a pivotal influence on CCA $(21,22)$.

Of the 16 DE IncRNAs identified in the ceRNA network, the complementary mRNAs mainly bound to H19, F-box and leucine rich repeat protein 19-AS1, HLA complex group 18, PVT1 and small nucleolar RNA host gene 1. H19 and PVT1 are the most widely studied lncRNAs among the $16 \mathrm{DE}$ lncRNAs. A growing number of studies have highlighted the fact that the oncofetal lncRNA gene H19 is a critical factor in embryonic development, fibrosis and tumorigenesis $(23,24)$. Accumulating evidence has revealed that the tumor suppressor protein and cell cycle regulator p53 negatively regulates $\mathrm{H} 19$ in tumor cells. Yang et al (25) reported that H19 was associated with p53 inactivation, which may contribute to suppression of apoptosis and increased cell proliferation in gastric cancer. Furthermore, it was reported that H19-derived miR-675 has an important role in inhibiting p53 and p53-dependent protein expression in bladder cancer cells in vivo (26). H19 also has crucial roles in tumor metastasis through the regulation of epithelial to mesenchymal transition (EMT), by functioning as an miRNA sponge in colorectal cancer $(27,28)$. A study has indicated that oxidative stress caused by infection is linked 

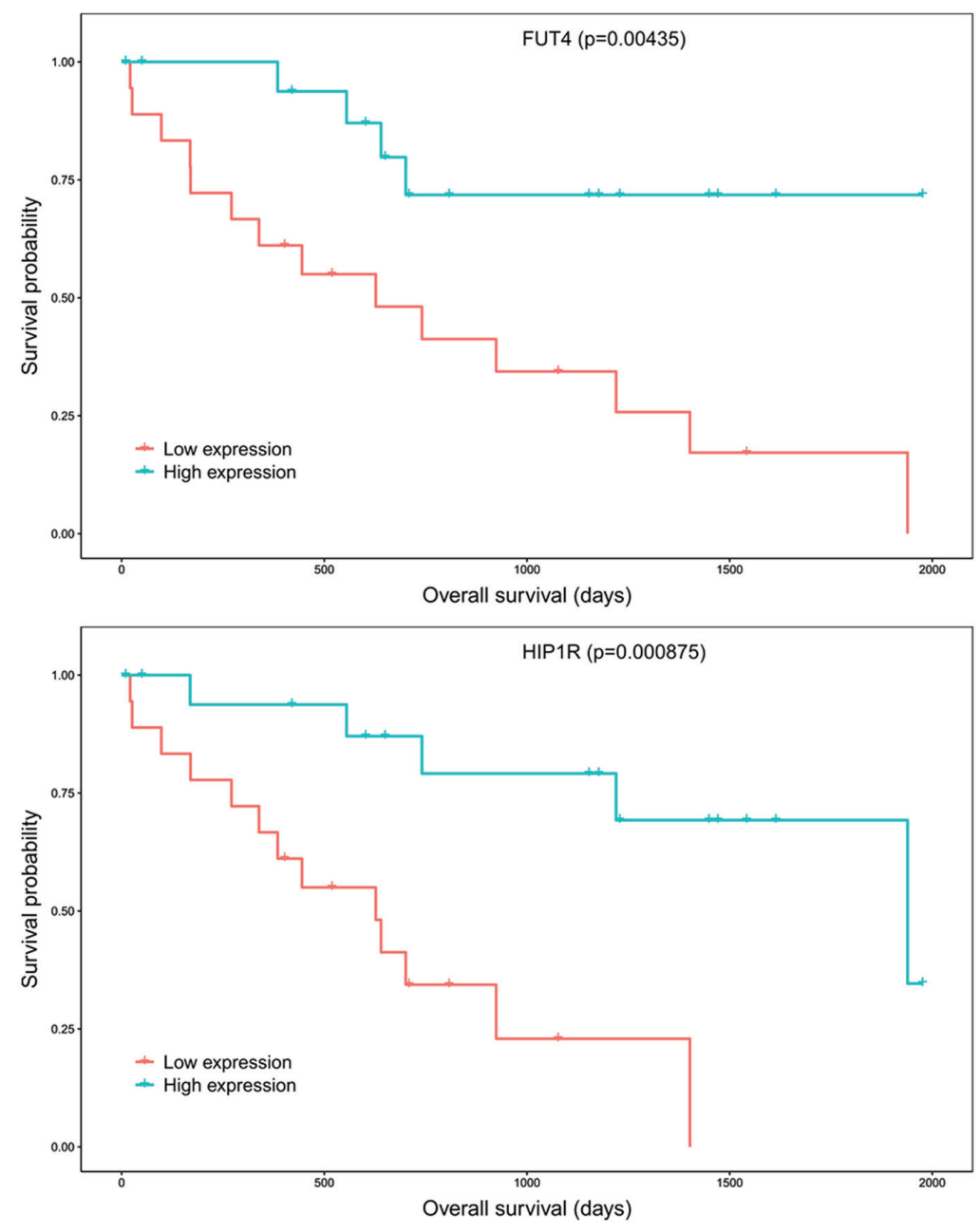

Figure 5. Kaplan-Meier analysis of overall survival associated with the expression status of the mRNAs FUT4 and HIP1R from the competing endogenous RNA network of cholangiocarcinoma patients. Other RNAs in the network analyzed using K-M methods with $\mathrm{P}<0.05$ were not shown in the figure. FUT4, fucosyltransferase 4; HIP1R, huntingtin-interacting protein 1 related.

to inflammation and the occurrence of CAA (29). In addition, H19 is considered to be involved in the progression of CCA by causing partial inactivation of interleukin-6 and downstream inflammatory responses triggered by oxidative stress (30). Similar to H19, overexpression of PVT1 has also been indicated to be associated with poor prognosis in a variety of human malignancies (31). In non-small cell lung cancer, PVT1 contributes to lung adenocarcinoma cell proliferation, and knockdown markedly reduces cell proliferation and induces apoptosis in vitro and in vivo (32). This phenomenon may be partly mediated through enhancer of zeste 2 polycomb repressive complex 2 subunit-associated suppression of the large tumor suppressor kinase 2/MDM2/p53 pathway. PVT1 has been further observed to be involved in the regulation of EMT, which is a vital step in the progression, invasion and metastasis of esophageal cancer (33). Furthermore, PVT1 was reported to promote liver fibrosis by downregulating patched 1 expression via binding to miR-152 and contributing to EMT (34). Huang et al (35) reported that increased expression of PVT1 is associated with tumor progression, and suggested that PVT1 may be an independent prognostic factor for poor prognosis in pancreatic cancer patients. Furthermore, a meta-analysis reported that increased PVT1 expression was significantly associated with factors of poor prognosis, including positive lymph node metastasis, positive distant metastasis, advanced tumor-nodes-metastasis stage and poor degree of differentiation, suggesting that PVT1 may be a potential biomarker in various cancer types (36). However, studies on the association between H19 or PVT1 and CCA have rarely been performed.

PPARs belong to the ligand-inducible nuclear hormone receptor superfamily (37). By forming heterodimers with retinoid $\mathrm{X}$ receptor, PPARs modulate the expression of lipid metabolism-, adipogenesis-, inflammation- and anti-cancer-associated genes in various human cancer types (37). In the present study, pathway analysis revealed that the PPAR signaling pathway was significantly enriched and exerted a pivotal influence in patients with CCA. Of all PPAR isoforms, PPAR $\gamma$ is considered to be most associated with tumors through the activation of different pathways. PPAR ligands have been reported to promote differentiation and apoptosis in numerous malignancies, including CCA, breast cancer and ovarian cancer (38-40). Han et al (41) reported that ligands of PPAR $\gamma$ inhibit the proliferation of CCA cells through p53-dependent mechanisms. Furthermore, the PPAR $\gamma$ ligand 15-deoxy- $\delta$-12,14-PGJ2 may induce apoptosis in CCA cell lines, although apoptosis-associated protein expression varies between different cell lines (41). In addition, accumulating 
evidence suggests that the mechanisms of the EMT promote the occurrence of CCA (42). PPAR $\gamma$ is able to upregulate the expression of Sprouty 4 through Wnt7A/Fzs9 signaling, and suppresses EMT (42). On the contrary, ectopic PPAR $\gamma$ expression in CCA cell lines may promote cell proliferation via the Smad pathway, which is a vital step in EMT $(43,44)$. Suzuki et al $(45)$ reported on the administration of the PPAR $\gamma$ agonist pioglitazone in a 73-year-old male patient diagnosed with diabetes, CCA and CCA-induced cholangiohepatitis. In this case, not only the diabetes was controlled, but also the cholangiohepatitis, which was thought to be linked to CCA progression. Furthermore, Asukai et at (46) reported that pioglitazone had a synergistic effect with gemcitabine and alleviated gemcitabine resistance in CCA gemcitabine-resistant cells by reducing miR-130a-3p expression in vitro. However, further studies of how PPARs are involved in CCA pathogenesis will be required.

The prognostic value of the ceRNA network was also analyzed. The results revealed that high expression of FUT4 $(\mathrm{P}<0.005)$ and HIP1R $(\mathrm{P}<0.001)$ was positively correlated with overall survival. However, according to previous studies, increased FUT4 expression was observed to be associated with poor prognosis in breast cancer, lung adenocarcinoma and colorectal cancer, which was contrary to the present results (47-49). Another study suggested that lower HIP1R protein expression is associated with a poor overall survival rate and progression-free survival in patients with diffuse large B-cell lymphoma (50). However, the opposite appears to be the case for prostate cancer: HIP1R was reported to increase the migratory and invasive properties of prostate cancer cells, and this may be suppressed by the miRNA-23b/27b cluster (51). To the best of our knowledge, the roles of FUT4 or HIP1R in CCA have not been assessed by any previous study.

The present study had several limitations. In the present study, only the data from TCGA database were considered to create the network. Furthermore, the network was not validated by any other database or in vitro analysis. Therefore, further confirmation of key RNAs and investigation of the possible molecular biological mechanisms of CCA in human CCA tissues will be performed in the future. Furthermore, the ceRNA network established in the present study requires broad validation by future studies.

In conclusion, in the present study, an integrated analysis of DE lncRNAs, miRNAs and mRNAs in CCA as performed and a ceRNA network was constructed. This ceRNA network may provide novel insight for further mechanistic investigation and may lead to improvements in the survival and prognosis of patients with CAA. Further experimental verification is required.

\section{Acknowledgements}

The authors would like to thank Professor Qulian Guo, Department of Anesthesia, Xiangya Hospital, Central South University, Changsha, Hunan, for his encouragement and guidance.

\section{Funding}

This study was supported by the National Science Foundation for Young Scientists of China (grant no. 81700275).

\section{Availability of data and materials}

All data were collected from The Cancer Genome Atlas (TCGA, https://portal.gdc.cancer.gov/)

\section{Authors' contributions}

WG concieved and designed the study. FX designed the experiments and wrote the first draft of the manuscript. YZ participated in data analysis, intepreted the data and revised the manuscript. GQ collected and analyzed the data. YH and LL prepared all figures and tables; WG and LL revised the manuscript and approved the final version. All authors reviewed the manuscript.

\section{Ethics approval and consent to participate}

Not applicable.

\section{Patient consent for publication}

Not applicable.

\section{Competing interests}

The authors declare that they have no competing interests.

\section{References}

1. Cai JQ, Cai SW, Cong WM and Chen MS: Diagnosis and treatment of cholangiocarcinoma: A consensus from surgical specialists of China. J Huazhong Univ Sci Technolog Med Sci 34: 469-475, 2014.

2. Ponting CP, Oliver PL and Reik W: Evolution and functions of long noncoding RNAs. Cell 136: 629-641, 2009.

3. Salmena L, Poliseno L, Tay Y, Kats L and Pandolfi P: A ceRNA hypothesis: The Rosetta stone of a hidden RNA language? Cell 146: 353-358, 2011.

4. Spizzo R, Almeida MI, Colombatti A and Calin GA: Long non-coding RNAs and cancer: A new frontier of translational research? Oncogene 31: 4577-4587, 2012.

5. Yang Z, Zhou L, Wu LM, Lai MC, Xie HY, Zhang F and Zheng SS: Overexpression of long non-coding RNA HOTAIR predicts tumor recurrence in hepatocellular carcinoma patients following liver transplantation. Ann Surg Oncol 18: 1243-1250, 2011.

6. Zhou X, Liu J and Wang W: Construction and investigation of breast-cancer-specific ceRNA network based on the mRNA and miRNA expression data. Let Syst Biol 8: 96-103, 2014.

7. Arun K, Arunkumar G, Bennet D, Chandramohan SM, Murugan AK and Munirajan AK: Comprehensive analysis of aberrantly expressed 1ncRNAs and construction of ceRNA network in gastric cancer. Oncotarget 9: 18386-18399, 2018.

8. Zhou M, Diao Z, Yue X, Chen Y, Zhao H, Cheng L and Sun J: Construction and analysis of dysregulated lncRNA-associated ceRNA network identified novel lncRNA biomarkers for early diagnosis of human pancreatic cancer. Oncotarget 7 : 56383-56394, 2016.

9. Peng H, Lu M and Selaru FM: The genome-wide gene expression profiling to predict competitive endogenous RNA network in hepatocellular cancer. Genomics Data 4: 93-95, 2015.

10. Shi X, Zhang H, Wang M, Xu X, Zhao Y,He R, Zhang M, Zhou M, Li X, Peng F, et al: LncRNA AFAP1-AS1 promotes growth and metastasis of cholangiocarcinoma cells. Oncotarget 8: 58394-58404, 2017.

11. Zhang S, Xiao J, Chai Y, Du YY, Liu Z, Huang K, Zhou X and Zhou W: LncRNA-CCAT1 promotes migration, invasion, and EMT in intrahepatic cholangiocarcinoma through suppressing miR-152. Dig Dis Sci 62: 3050-3058, 2017. 
12. Chan WL, Huang HD and Chang JG: lncRNAMap: A map of putative regulatory functions in the long non-coding transcriptome. Comput Biol Chem 50: 41-49, 2014.

13. Robinson MD, McCarthy DJ and Smyth GK: edgeR: A Bioconductor package for differential expression analysis of digital gene expression data. Bioinformatics 26: 139-140, 2010.

14. Ashwini J, Marks DS and Erik L: miRcode: A map of putative microRNA target sites in the long non-coding transcriptome. Bioinformatics 28: 2062-2063, 2012.

15. Chou CH, Shrestha S, Yang CD, Chang NW, Lin YL, Liao KW, Huang WC, Sun TH, Tu SJ, Lee WH, et al: miRTarBase update 2018: A resource for experimentally validated microRNA-target interactions. Nucleic Acids Res 46: D296-D302, 2018.

16. Wong $\mathrm{N}$ and Wang X: miRDB: An online resource for microRNA target prediction and functional annotations. Nucleic Acids Res 43: D146-D152, 2015.

17. Agarwal V, Bell GW, Nam JW and Bartel DP: Predicting effective microRNA target sites in mammalian mRNAs. Elife 4, 2015

18. Li YY,Li H,Lv P, Liu G, Li XR, Tian BN and Chen DJ: Prognostic value of cirrhosis for intrahepatic cholangiocarcinoma after surgical treatment. J Gastrointest Surg 15: 608-613, 2011.

19. Batista PJ and Chang HY: Long Noncoding RNAs: Cellular address codes in development and disease. Cell 152: 1298-1307, 2013.

20. Zheng B, Jeong S, Zhu Y, Chen L and Xia Q: miRNA and lncRNA as biomarkers in cholangiocarcinoma(CCA). Oncotarget 8: 100819-100830, 2017.

21. Huang QX, Cui JY, Ma H, Jia XM, Huang FL and Jiang LX: Screening of potential biomarkers for cholangiocarcinoma by integrated analysis of microarray data sets. Cancer Gene Ther 23 : 48-53, 2016.

22. Yang W, Li Y, Song X, Xu J and Xie J: Genome-wide analysis of long noncoding RNA and mRNA co-expression profile in intrahepatic cholangiocarcinoma tissue by RNA sequencing. Oncotarget 8: 26591-26599, 2017.

23. Sun H, Wang G, Peng Y,Zeng Y,Zhu QN, Li TL, Cai JQ, Zhou HH and Zhu YS: H19 lncRNA mediates 17 $\beta$-estradiol-induced cell proliferation in MCF-7 breast cancer cells. Oncol Rep 33: 3045-3052, 2015.

24. Li L, Chen W, Wang Y, Tang L and Han M: Long non-coding RNA H19 regulates viability and metastasis, and is upregulated in retinoblastoma. Oncol Lett 15: 8424-8432, 2018.

25. Yang F, Bi J, Xue X, Zheng L, Zhi K, Hua J and Fang G: Up-regulated long non-coding RNA H19 contributes to proliferation of gastric cancer cells. FEBS J 279: 3159-3165, 2012

26. Liu C, Chen Z, Fang J, Xu A, Wei Z and Wang Z: H19-derived miR-675 contributes to bladder cancer cell proliferation by regulating p53 activation. Tumor Biol 5: 263-270, 2016.

27. Liang WC, Fu WM, Wong CW, Wang Y, Wang WM, Hu GX, Zhang L, Xiao LJ, Wan DC, Zhang JF and Waye MM: The IncRNA H19 promotes epithelial to mesenchymal transition by functioning as miRNA sponges in colorectal cancer. Oncotarget 6: 22513-22525, 2015.

28. Xu Y, Wang Z, Jiang X and Cui Y: Overexpression of long noncoding RNA H19 indicates a poor prognosis for cholangiocarcinoma and promotes cell migration and invasion by affecting epithelial-mesenchymal transition. Biomed Pharmacother 92: 17-23, 2017.

29. Boonyanugomol W, Chomvarin C, Sripa B, Bhudhisawasdi V, Khuntikeo N, Hahnvajanawong $\mathrm{C}$ and Chamsuwan $\mathrm{A}$ Helicobacter pylori in Thai patients with cholangiocarcinoma and its association with biliary inflammation and proliferation. HPB (Oxford) 14: 177-184, 2012.

30. Wang WT, Ye H, Wei PP, Han BW, He B, Chen ZH and Chen YQ: LncRNAs H19 and HULC, activated by oxidative stress, promote cell migration and invasion in cholangiocarcinoma through a ceRNA manner. J Hematol Oncol 9: 117, 2016.

31. Zhou DD, Liu XF, Lu CW, Pant OP and Liu XD: Long non-coding RNA PVT1: Emerging biomarker in digestive system cancer. Cell Prolif 50: e12398, 2017.

32. Wan L, Sun M, Liu GJ, Wei CC, Zhang EB, Kong R, Xu TP, Huang MD and Wang ZX: Long non-coding RNA PVT1 promotes non-small cell lung cancer cell proliferation through epigenetically regulating LATS2 expression. Mol Cancer Ther 15: 1082-1094, 2016.

33. Shuang Y, Ning Q, Zhang G, Hong S, Zhen W and Li Y: Construction of differential mRNA-lncRNA crosstalk networks based on ceRNA hypothesis uncover key roles of lncRNAs implicated in esophageal squamous cell carcinoma. Oncotarget 7: 85728-85740, 2016
34. Zheng J, Yu F, Dong P, Wu L, Zhang Y, Hu Y and Zheng L: Long non-coding RNA PVT1 activates hepatic stellate cells through competitively binding microRNA-152. Oncotarget 7: 62886-62897, 2016.

35. Huang C, Yu W, Wang Q, Cui H, Wang Y, Zhang L, Han F and Huang T: Increased expression of the lncRNA PVT1 is associated with poor prognosis in pancreatic cancer patients. Minerva Med 106: 143-149, 2015.

36. Lu D, Luo P, Wang Q, Ye Y and Wang B: IncRNA PVT1 in cancer: A review and meta-analysis. Clin Chim Acta 474: 1-7, 2017.

37. Semple RK, Chatterjee VK and O'Rahilly S: PPAR gamma and human metabolic disease. J Clin Invest 116: 581-589, 2006.

38. Colincassin C, Yao X, Cerella C, et al: PPAR, breast cancer. Molecular Carcinogenesis 54: 393-404, 2013.

39. Morgado $M$ and Carson DD: PPAR $\gamma$ Modulation of cytokine-stimulated MUC16 (CA125) expression in breast and ovarian cancer-derived cells. J Cell Biochem 118: 163-171, 2016.

40. Xu D, Davis BB, Wang ZH, Zhao SP, Wasti B, Liu ZL, Li N, Morisseau $\mathrm{C}$, Chiamvimonvat $\mathrm{N}$ and Hammock BD: A potent soluble epoxide hydrolase inhibitor, t-AUCB, acts through PPAR $\gamma$ to modulate the function of endothelial progenitor cells from patients with acute myocardial infarction. Int J Cardiol 167: 1298-1304, 2013.

41. Han C, Demetris AJ, Michalopoulos GK, Zhan Q, Shelhamer JH and Wu T: PPARgamma ligands inhibit cholangiocarcinoma cell growth through p53-dependent GADD45 and p21 pathway. Hepatology 38: 167-177, 2003.

42. Tennis MA, Van Scoyk MM, Freeman SV, Vandervest KM, Nemenoff RA and Winn RA: Sprouty-4 inhibits transformed cell growth, migration and invasion, and epithelial-mesenchymal transition, and Is Regulated by Wnt7A through PPARgamma in non-small cell lung cancer. Mol Cancer Res 8: 833-843, 2010

43. Han C, Demetris AJ,Liu Y, ShelhamerJH and Wu T: Transforming growth factor- $\beta$ (TGF- $\beta$ ) activates cytosolic phospholipase A2 $\alpha$ $(\mathrm{cPLA} 2 \alpha)$-mediated prostaglandin E2 (PGE)2/EP1 and peroxisome proliferator-activated receptor- $\gamma(\mathrm{PPAR}-\gamma) / \mathrm{Smad}$ signaling pathways in human liver cancer cells. JBC: Aug 4, 2004 (Epub ahead of print). doi: 10.1074/jbc.M404852200.

44. Reka AK, Kurapati H, Narala VR, Bommer G, Chen J, Standiford TJ and Keshamouni VG: Peroxisome proliferator-activated receptor-gamma activation inhibits tumor metastasis by antagonizing Smad3-mediated epithelial-mesenchymal transition. Mol Cancer Ther 9: 3221-3232, 2010.

45. Suzuki S, Mori J, Yamazaki M, Sato A, Hosoda W and Hashizume K: Beneficial effects of pioglitazone on cholangiohepatitis induced by bile duct carcinoma. Internal Med 46: 1723-1727, 2007.

46. Asukai K, Kawamoto K, Eguchi H, Konno M, Asai A, Iwagami Y, Yamada D, Asaoka T, Noda T, Wada H, et al: Micro-RNA-130a-3p Regulates Gemcitabine resistance via PPARG in cholangiocarcinoma. Ann Surg Oncol 24: 2344-2352, 2017.

47. Nagaraj NS and Singh OV: Integrating genomics and proteomicsoriented biomarkers to comprehend lung cancer. Expert Opin Med Diagn 3: 167-180, 2009.

48. Wang A, Lu C, Ning Z, Gao W, Xie Y, Zhang N, Liang J, Abbasi FS, Yan Q and Liu J: Tumor-associated macrophages promote Ezrin phosphorylation-mediated epithelial-mesenchymal transition in lung adenocarcinoma through FUT4/LeY up-regulation. Oncotarget 8: 28247-28259, 2017.

49. Li Y, Sun Z, Liu B, Shan Y, Zhao L and Jia L: Tumor-suppressive miR-26a and miR-26b inhibit cell aggressiveness by regulating FUT4 in colorectal cancer. Cell Death Dis 8: e2892, 2017.

50. Wong KK, Gascoyne DM, Brown PJ, Soilleux EJ, Snell C, Chen H, Lyne L, Lawrie CH, Gascoyne RD, Pedersen LM, et al: Reciprocal expression of the endocytic protein HIP1R and its repressor FOXP1 predicts outcome in R-CHOP-treated diffuse large B-cell lymphoma patients. Leukemia 28: 362-372, 2014.

51. Rice MA, Ishteiwy RA, Magani F, Udayakumar T, Reiner T, Yates TJ, Miller P, Perez-Stable C, Rai P, Verdun R, et al: The microRNA-23b/-27b cluster suppresses prostate cancer metastasis via Huntingtin-interacting protein 1-related. Oncogene 35: 4752-4761, 2016. 